\title{
Pristimerin is a Promising Natural Product against Breast Cancer in vitro and in vivo through Apoptosis and the Blockage of Autophagic Flux ${ }^{\dagger}$
}

\author{
Buse Cevatemre ${ }^{1}$, Konstantinos Dimas ${ }^{2}$, Bruno Botta ${ }^{3}$ and Engin Ulukaya ${ }^{4, *}$ \\ 1 Department of Biology, Faculty of Arts and Sciences, Uludag University, Bursa 16059, Turkey; \\ bcevatemre@uludag.edu.tr \\ 2 Department of Pharmacology, Faculty of Medicine, University of Thessaly, 41222 Larissa, Greece; \\ ksdimas@yahoo.com \\ 3 Dipartimento di Chimica e Tecnologie del Farmaco, Sapienza University of Roma, piazzale Aldo Moro 5, \\ 00185 Roma, Italy; bruno.botta@uniroma1.it \\ 4 Department of Clinical Biochemistry, Faculty of Medicine, Istinye University, Istanbul 34010, Turkey \\ * Correspondence: eulukaya@istinye.edu.tr; Tel.: +90-533-430-932 \\ + Presented at the 2nd International Conference on Natural Products for Cancer Prevention and Therapy, \\ Kayseri, Turkey, 8-11 November 2017.
}

Published: 15 November 2017

\begin{abstract}
The exploration of natural products is of importance because the management of cancer is still not satisfactory. Pristimerin is a naturally occurring triterpenoid that has been shown to suppress the proliferation of various cancer cell lines at relatively lower concentrations, of which the IC50 values are around $0.5-4 \mu \mathrm{M}$. Its cytotoxic potential on particularly cancer stem cells (CSCs) should be much more important due to the CSCs' recent role in recurrence of cancer. Although pristimerin exerted growth inhibitory activity in various cancer cells, the mechanisms is still debatable. Its antigrowth effect seems to involve the blockage of autophagic process as well. In our studies, pristimerin showed an anti-growth effect on cancer cells and cancer stem cells with IC50 values ranging at $0.38-1.75 \mu \mathrm{M}$. Apoptosis was induced in MCF-7 and MCF-7s (CSCs) cells. It also resulted in an incompleted autophagy as evidenced by the increase of autophagy-related proteins (P62 and LC3-II) as well as an unfolded protein response (UPR). Moreover, it inhibited the growth of xenografts in NOD/SCID mice. Taken together, pristimerin deserves further attention in terms of its use for the treatment of breast cancer. In this talk, quite an unusal cytotoxic potential of pristimerin will be discussed.
\end{abstract}

Keywords: pristimerin; autophagic flux; NOD/SCID mice

Conflicts of Interest: The authors declare no conflict of interest.

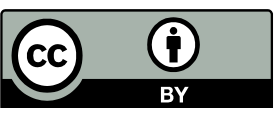

(C) 2017 by the authors. Licensee MDPI, Basel, Switzerland. This article is an open access article distributed under the terms and conditions of the Creative Commons Attribution (CC BY) license (http://creativecommons.org/licenses/by/4.0/). 